\title{
Log-Domain Calculation of the 1959 Sphere-Packing Bound with Application to M-ary PSK Block Coded Modulation
}

\author{
Igal Sason Gil Wiechman \\ Department of Electrical Engineering \\ Technion - Israel Institute of Technology \\ Haifa 32000, Israel \\ Email: \{sason@ee, igillw@tx\}.technion.ac.il
}

\begin{abstract}
The 1959 sphere-packing bound of Shannon is reviewed, and a new algorithm is derived for its calculation. This algorithm performs the entire calculation in the logarithmic domain, thus facilitating the exact calculation of the bound for moderate to large block lengths. The new algorithm circumvents the numerical difficulties associated with a previously suggested algorithm by Valembois and Fossorier. We also compare the bound with a new sphere-packing bound which was recently derived by the authors. By applying the bound to M-ary PSK block coded modulation, the new bound suggests an interesting alternative to the Shannon bound.
\end{abstract}

\section{INTRODUCTION}

Error correcting codes which employ efficient iterative decoding algorithms closely approach the capacity limit of many standard communication channels. The performance of these codes is especially appealing for moderate to large block lengths. These developments stirred up new interest in studying the limits of code performance as a function of the block length (see, e.g., [1]-[5] and [8]-[11]).

Sphere-packing bounds are lower bounds on the errorprobability of block codes in terms of the rate, block length and communication channel. These bounds are valid under maximum-likelihood (ML) decoding or in general under list decoding. Classical members of this family are the 1959 sphere-packing (SP59) bound of Shannon [6] which refers to the AWGN channel and the 1967 sphere-packing (SP67) bound of Shannon, Gallager and Berlekamp [7] which is valid for any discrete memoryless channel. The latter bound has been recently improved by Valembois and Fossorier [8] and Wiechman and Sason [10]. These lower bounds on the decoding error probability are often used as a reference for quantifying the sub-optimality of codes with their practical decoding algorithms; by comparing computer simulations for the performance of turbo-like codes over a wide range of rates and block sizes, it was exemplified in the literature that the gap between the sphere-packing bounds and the performance of these codes under efficient iterative decoding algorithms can be reduced below $1 \mathrm{~dB}$.

The calculation of the SP59 bound is numerically involved and becomes prohibitively complex when the block length exceeds several hundreds. To overcome this problem, Shannon provides in [6] some asymptotic approximations for the SP59 bound which enable to estimate of value of the bound for large block lengths. These approximations perform the calculation in the logarithmic domain, thus circumventing the numerical problems which hinder the exact calculation of the bound. In [8], a recursive algorithm for the calculation of the SP59 bound is presented. This algorithm performs the calculations in the natural domain and is therefore prone to numerical over and underflows when applied to block lengths of more than several hundreds.

This paper presents an algorithm which performs the entire calculation of the SP59 bound in the logarithmic domain. This technique allows us to calculate the bound for all block lengths without encountering numerical difficulties. The new algorithm is applied to calculate the SP59 bound for several moderate to large block lengths and compare the exact value of the bound with the asymptotic approximations in [6]. This algorithm is part of a more general study of sphere-packing bounds in [10]. The second part of this study is the derivation of an improved sphere-packing (ISP) bound which, under a mild condition, is valid for general memoryless channels; this bound was recently presented in the conference paper [11]. The ISP bound is based on the work in [7] and [8] and improves the tightness of the bounds derived in these works.

The paper is structured as follows: Section II reviews the SP59 bound of Shannon and the asymptotic approximations provided in [6]. Section III presents the recursive algorithm for the calculation of the SP59 bound, as derived in [8]. The numerical instability of this algorithm motivates the derivation of a new algorithm for calculating the bound in the logarithmic domain which is performed in Section IV. Section V presents a short discussion of the ISP bound presented in [10], [11] along with some numerical results comparing the SP59 and ISP bounds for M-ary PSK signaling over the AWGN channel. The paper is summarized in Section VI. Some technical calculations are relegated to an Appendix. For a comprehensive tutorial review of classical sphere-packing bounds (i.e., the SP59 and SP67 bounds) and recent improvements in [8], the reader is referred to [5, Chapter 5].

\section{The 1959 SPHERE-PACKING Bound AND ASYMPTOTIC APPROXIMATIONS}

The SP59 bound of Shannon [6] provides a lower bound on the decoding error probability of block codes transmitted over the AWGN channel. Consider a block code $\mathcal{C}$ of length $N$, and rate $R$ nats per channel use per dimension. It is 
assumed that the codewords are mapped to equal energy signals (e.g., PSK modulation); hence, each transmitted signal corresponds to a point an $N$-dimensional sphere centered at the origin, but finer details of the modulation are not considered. This assumption implies that every Voronoi cell (i.e., the convex region containing all the points which are closer to the considered signal than any other code signal) is a polyhedric cone limited by at most $\exp (N R)-1$ hyper planes intersecting at the origin. To measure the size of the Voronoi regions, Shannon introduced the solid angle of a cone which is defined to be the area of the sphere of unit radius which is cut out by the cone. Since the Voronoi cells partition the space $\mathbb{R}^{N}$, the sum of their solid angles must be the area of an $N$-dimensional sphere of unit radius. The derivation of the SP59 bound relies on two main observations:

- Among the cones of a given solid angle, the lowest error probability is achieved by the circular cone whose axis connects the code signal with the origin.

- It is best to share the total solid angle equally among the $\exp (N R)$ Voronoi regions.

From these observations it follows that the average Voronoi cell of any code cannot be better than a circular cone with a solid angle equal to $\exp (-N R)$ of the solid angle of $\mathbb{R}^{N}$ centered around the code signal. The solid angle of a circular cone is given by the following lemma.

Lemma 1 (Solid Angle of a Circular Cone [6]): The solid angle of a circular cone of half angle $\theta$ in $\mathbb{R}^{N}$ is given by

$$
\Omega_{N}(\theta)=\frac{2 \pi^{\frac{N-1}{2}}}{\Gamma\left(\frac{N-1}{2}\right)} \int_{0}^{\theta}(\sin \phi)^{N-2} d \phi .
$$

In particular, the solid angle of $\mathbb{R}^{N}$ is

$$
\Omega_{N}(\pi)=\frac{2 \pi^{\frac{N}{2}}}{\Gamma\left(\frac{N}{2}\right)} .
$$

Theorem 1 (The 1959 Sphere-Packing (SP59) Bound [6]): Assume that a block code of length $N$ and rate $R$ nats per dimension is transmitted over an AWGN channel with noise spectral density $\frac{N_{0}}{2}$. Then, under ML decoding, the error probability satisfies

$$
P_{\mathrm{e}}(\mathrm{ML})>P_{\mathrm{SPB}}(N, \theta, A), \quad A \triangleq \sqrt{\frac{2 E_{\mathrm{s}}}{N_{0}}}
$$

where $E_{\mathrm{s}}$ is the average energy per code symbol, $\theta \in[0, \pi]$ satisfies the inequality $2^{-N R} \leq \frac{\Omega_{N}(\theta)}{\Omega_{N}(\pi)}$,

$$
\begin{aligned}
P_{\mathrm{SPB}}(N, \theta, A) \triangleq \quad & Q(\sqrt{N} A)+\frac{(N-1) e^{-\frac{N A^{2}}{2}}}{\sqrt{2 \pi}} \\
& \cdot \int_{\theta}^{\frac{\pi}{2}}(\sin \phi)^{N-2} f_{N}(\sqrt{N} A \cos \phi) d \phi(1)
\end{aligned}
$$

and

$$
f_{N}(x) \triangleq \frac{1}{2^{\frac{N-1}{2}} \Gamma\left(\frac{N+1}{2}\right)} \int_{0}^{\infty} z^{N-1} \exp \left(-\frac{z^{2}}{2}+z x\right) d z
$$

where $x \in \mathbb{R}$ and $N \in \mathbb{N}$.

By assumption, the transmitted signal corresponds to point on the $N$-dimensional sphere of radius $\sqrt{N E_{s}}$ centered at the origin. The value $P_{\mathrm{SPB}}(N, \theta, A)$ in the RHS of (1) designates the probability that the additive Gaussian noise carries the transmitted signal outside the $N$-dimensional circular cone of half angle $\theta$ whose main axis connects the origin and the transmitted signal point. Hence, this function is monotonically decreasing in $\theta$. The tightest lower bound on the decoding error probability is therefore achieved for $\theta_{1}(N, R)$ which satisfies

$$
\frac{\Omega_{N}\left(\theta_{1}(N, R)\right)}{\Omega_{N}(\pi)}=\exp (-N R) .
$$

Solving this equation to find the optimal angle $\theta_{1}(N, R)$ is a tedious task which considerably increases the computational complexity of the evaluating the SP59 bound. To simplify the calculation of the bound, [6] provides asymptotically tight upper and lower bounds on the ratio $\frac{\Omega_{N}(\theta)}{\Omega_{N}(\pi)}$.

Lemma 2 (Bounds on the Solid Angle [6]): The solid angle of a circular cone of half angle $\theta$ in the Euclidean space $\mathbb{R}^{N}$ satisfies the inequality

$\frac{\Gamma\left(\frac{N}{2}\right)(\sin \theta)^{N-1}}{2 \Gamma\left(\frac{N+1}{2}\right) \sqrt{\pi} \cos \theta}\left(1-\frac{\tan ^{2} \theta}{N}\right) \leq \frac{\Omega_{N}(\theta)}{\Omega_{N}(\pi)} \leq \frac{\Gamma\left(\frac{N}{2}\right)(\sin \theta)^{N-1}}{2 \Gamma\left(\frac{N+1}{2}\right) \sqrt{\pi} \cos \theta}$.

Corollary 1 (SP59 Bound (Cont.)): If $\theta^{*}$ satisfies

$$
\frac{\Gamma\left(\frac{N}{2}\right)\left(\sin \theta^{*}\right)^{N-1}}{2 \Gamma\left(\frac{N+1}{2}\right) \sqrt{\pi} \cos \theta^{*}}\left(1-\frac{\tan ^{2} \theta^{*}}{N}\right)=\exp (-N R)
$$

then $\frac{\Omega_{N}\left(\theta^{*}\right)}{\Omega_{N}(\pi)} \geq \exp (-N R)$, and therefore

$$
P_{\mathrm{e}}(\mathrm{ML})>P_{\mathrm{SPB}}\left(N, \theta^{*}, A\right) .
$$

The use of $\theta^{*}$ instead of the optimal value $\theta_{1}(N, R)$ causes some loss in the tightness of the SP59 bound. However, due to the asymptotic tightness of the bounds on $\frac{\Omega_{N}(\theta)}{\Omega_{N}(\pi)}$, the loss in the tightness of the bound in Corollary 1 vanishes asymptotically as $N \rightarrow \infty$. In [8], it was numerically observed that the loss is marginal even for relatively small values of $N$ and $R$.

The calculation of the SP59 bound is numerically challenging and causes over and under flows in the floating point operations when the block length exceeds several hundreds; increasing the precision of the floating point numbers does little to increase the range where the bound can be calculated. To overcome this, [6] provides some asymptotic formulas which give an accurate estimation of the bound when the block lengths become very large. These approximations allow the calculation to be made in the logarithmic domain which virtually eliminates the possibility of floating point errors.

Theorem 2: [6]: Defining

$$
\begin{aligned}
G(\theta) & \triangleq \frac{A \cos \theta+\sqrt{A^{2} \cos ^{2} \theta+4}}{2} \\
E_{L}(\theta) & \triangleq \frac{A^{2}-A G(\theta) \cos \theta-2 \ln (G(\theta) \sin \theta)}{2}
\end{aligned}
$$

then

$$
P_{\mathrm{SPB}}(N, \theta, A) \geq \frac{\sqrt{N-1}}{6 N(A+1)} e^{\frac{-(A+1)^{2}+3}{2}} e^{-N E_{L}(\theta)} .
$$

This lower bound is valid for any block length $N$. However, the ratio of the left and right terms in (5) stays bounded away from one for all $N$. A more accurate approximation of $P_{\mathrm{SPB}}(N, \theta, A)$ is given below, but without a determined inequality. As a consequence, the following approximation is 
not a proven theoretical lower bound on the error probability. For $N>1000$, however, its numerical values become very close to the exact value, giving a useful approximation of the SP59 bound. Following the notation of Theorem 2, [6] shows that if $\theta>\cot ^{-1}(A)$, then

$$
P_{\mathrm{SPB}}(N, \theta, A) \approx \frac{\alpha(\theta) e^{-N E_{L}(\theta)}}{\sqrt{N}}
$$

where

$$
\alpha(\theta) \triangleq\left(\sqrt{\pi\left(1+G(\theta)^{2}\right)} \sin \theta\left(A G(\theta) \sin ^{2} \theta-\cos \theta\right)\right)^{-1} .
$$

III. A Recursive Algorithm for CAlculating the 1959 SPHERE-PACKING BOUND [8]

In [8, Section 2], the SP59 bound is reviewed and a recursive algorithm to simplify its calculation is suggested. This algorithm is given by the following theorem:

Theorem 3 (Recursive Calculation of the SP59 Bound):

[8, Theorem 3]: The set of functions $\left\{f_{N}\right\}$ introduced in (2) can be expressed in the alternative form

$f_{N}(x)=P_{N}(x)+Q_{N}(x) \exp \left(\frac{x^{2}}{2}\right) \int_{-\infty}^{x} \exp \left(-\frac{t^{2}}{2}\right) d t, \quad \begin{aligned} & x \in \mathbb{R}, \\ & N \in \mathbb{N}\end{aligned}$

where $P_{N}$ and $Q_{N}$ are two polynomials, determined by the same recursive equation for all $N \geq 5$

$$
\begin{aligned}
& P_{N}(x)=\frac{2 N-5+x^{2}}{N-1} P_{N-2}(x)-\frac{N-4}{N-1} P_{N-4}(x), \\
& Q_{N}(x)=\frac{2 N-5+x^{2}}{N-1} Q_{N-2}(x)-\frac{N-4}{N-1} Q_{N-4}(x)
\end{aligned}
$$

with the initial conditions

$$
\begin{aligned}
& P_{1}(x)=0, \quad Q_{1}(x)=1 \\
& P_{2}(x)=\sqrt{\frac{2}{\pi}}, \quad Q_{2}(x)=\sqrt{\frac{2}{\pi}} x \\
& P_{3}(x)=\frac{x}{2}, \quad Q_{3}(x)=\frac{1+x^{2}}{2} \\
& P_{4}(x)=\sqrt{\frac{2}{\pi}} \frac{2+x^{2}}{3}, \quad Q_{4}(x)=\sqrt{\frac{2}{\pi}} \frac{3 x+x^{3}}{3} .
\end{aligned}
$$

Observing the recursive equations for $P_{N}$ and $Q_{N}$ in (7), we notice that the coefficients of the higher powers of $x$ vanish exponentially as $N$ increases. When performing the calculation using double-precision floating point numbers, these coefficients cause underflows when $N$ is larger than several hundreds, and are replaced by zeros. However, examining (1), we observe that the function $f_{N}(x)$ is typically evaluated at $x \sim O(\sqrt{N})$. Hence, as $N$ becomes larger than several hundreds, the underflows in the calculation of the coefficients for the higher powers of $X$ cause a considerable accuracy in the calculation of $P_{\mathrm{SPB}}$ in (1). For further discussion on the effect of these numerical difficulties the reader is referred to [10, Section 4.2]

\section{A Log-Domain Approach For COMputing the 1959 SPHERE-PACKING BOUND}

The numerical difficulties which makes the calculation of the SP59 bound intractable for moderate to large block lengths can be overcome by performing the calculation in the logarithmic domain. In this section, we present a method which performs the calculation of the integrand in the RHS of (1) in the log domain. This approach circumvents the numerical over and under flows which become problematic in the calculation of the SP59 bound for large block lengths. We begin our derivation by representing the set of functions $\left\{f_{N}\right\}$ defined in (2) as a sum of exponents.

Proposition 1: The set of functions $\left\{f_{N}\right\}$ in (2) can be expressed in the form

$$
f_{N}(x)=\sum_{j=0}^{N-1} \exp (d(N, j, x)), \quad x \in \mathbb{R}, N \in \mathbb{N}
$$

where for all $N \in \mathbb{N}, x \in \mathbb{R}$ and $j=0,1 \ldots, N-1$

$$
\begin{aligned}
d(N, j, x) \triangleq & \frac{x^{2}}{2}+\ln \Gamma\left(\frac{N}{2}\right)-\ln \Gamma\left(\frac{j}{2}+1\right)-\ln \Gamma(N-j) \\
& +(N-1-j) \ln (\sqrt{2} x)-\frac{\ln 2}{2} \\
& +\ln \left[1+(-1)^{j} \tilde{\gamma}\left(\frac{x^{2}}{2}, \frac{j+1}{2}\right)\right]
\end{aligned}
$$

and

$$
\begin{aligned}
\Gamma(a) & \triangleq \int_{0}^{\infty} t^{a-1} e^{-t} d t, \quad \operatorname{Re}(a)>0 \\
\tilde{\gamma}(x, a) & \triangleq \frac{1}{\Gamma(a)} \int_{0}^{x} t^{a-1} e^{-t} d t, \quad x \in \mathbb{R}, \operatorname{Re}(a)>0
\end{aligned}
$$

designate the complete and incomplete Gamma functions, respectively.

The proof of this proposition is given in the Appendix.

Remark 1: Note that the exponents $d(N, j, x)$ in (8) are readily calculated using standard mathematical functions. The function which calculates the natural logarithm of the Gamma function is implemented in the MATLAB software by gammaln, and in the Mathematica software by LogGamma. The function $\tilde{\gamma}(a, b)$ is implemented in MATLAB by gammainc $(x, N)$ and in Mathematica by GammaRegularized ( $, 0, x)$.

To complete the calculation of the function $f_{N}$ in the $\log$ domain, we employ the function

$$
\max ^{*}\left(x_{1}, \ldots, x_{m}\right) \triangleq \ln \left(\sum_{i=1}^{m} e^{x_{i}}\right), \quad \begin{gathered}
m \in \mathbb{N}, \\
x_{1}, \ldots, x_{m} \in \mathbb{R}
\end{gathered}
$$

which is commonly used in the implementation of the logdomain BCJR algorithm. The function $\max ^{*}$ is calculated in the $\log$ domain using the recursive equation

$$
\begin{array}{r}
\max ^{*}\left(x_{1}, \ldots, x_{m+1}\right)=\max ^{*}\left(\max ^{*}\left(x_{1}, \ldots, x_{m}\right), x_{m+1}\right), \\
m \in \mathbb{N} \backslash\{1\}, x_{1}, \ldots, x_{m+1} \in \mathbb{R}
\end{array}
$$

with the initial condition

$$
\max ^{*}\left(x_{1}, x_{2}\right)=\max \left(x_{1}, x_{2}\right)+\ln \left(1+e^{-\left|x_{1}-x_{2}\right|}\right) .
$$

Combining Proposition 1 and the definition of the function $\max ^{*}$ in (11), yields a log domain algorithm for the calculation of the set of functions $\left\{f_{N}\right\}$.

Corollary 2: The set of functions $\left\{f_{N}\right\}$ defined in (2) can be rewritten in the form

$$
f_{N}(x)=\exp \left[\max ^{*}(d(N, 0, x), d(N, 1, x), \ldots, d(N, N-1, x))\right]
$$

where $d(N, j, x)$ is introduced in (8). 
Substituting the log domain form of $f_{N}$ in the RHS of (1), we get an efficient algorithm for the calculation of the SP59 bound in the log domain; this algorithm is given in the following theorem:

Theorem 4 (Log domain calculation of the SP59 bound): The term $P_{\mathrm{SPB}}(N, \theta, A)$ in the RHS of (1) can be rewritten as

$$
\begin{aligned}
P_{\mathrm{SPB}}(N, \theta, A)= & \int_{\theta}^{\frac{\pi}{2}} \exp \left[\ln (N-1)-\frac{N A^{2}}{2}-\frac{1}{2} \ln (2 \pi)\right. \\
& +(N-2) \ln \sin \phi \\
& \left.\quad+\max ^{*}\left(\{d(N, i, \sqrt{N} A \cos \phi)\}_{i=0}^{N-1}\right)\right] d \phi \\
& +Q(\sqrt{N} A), \quad N \in \mathbb{N}, \theta \in\left[0, \frac{\pi}{2}\right], A \in \mathbb{R}^{+}
\end{aligned}
$$

where $d(N, j, x)$ is defined in (8).

Using Theorem 4, it is possible to calculate the exact value of the SP59 lower bound for very large block lengths. Figure 1 shows a comparison of the exact value of the SP59 bound and its asymptotic value as given in Theorem 4 and Eq. (6), respectively. This comparison is shown for a code rate of 0.5 bits per channel use per dimension and block lengths of $N=$ $10^{2}, 10^{3}$ and $10^{4}$. The calculations of the exact and asymptotic expressions were done using $\theta^{*}$ from (3); due to the large block lengths, the loss incurred by using this suboptimal value is negligible. It is observed that the asymptotic expression is indeed quite accurate for the two larger block lengths of $N=$ 1,000 and 10,000, and its accuracy is improved by increasing the block length and the SNR.

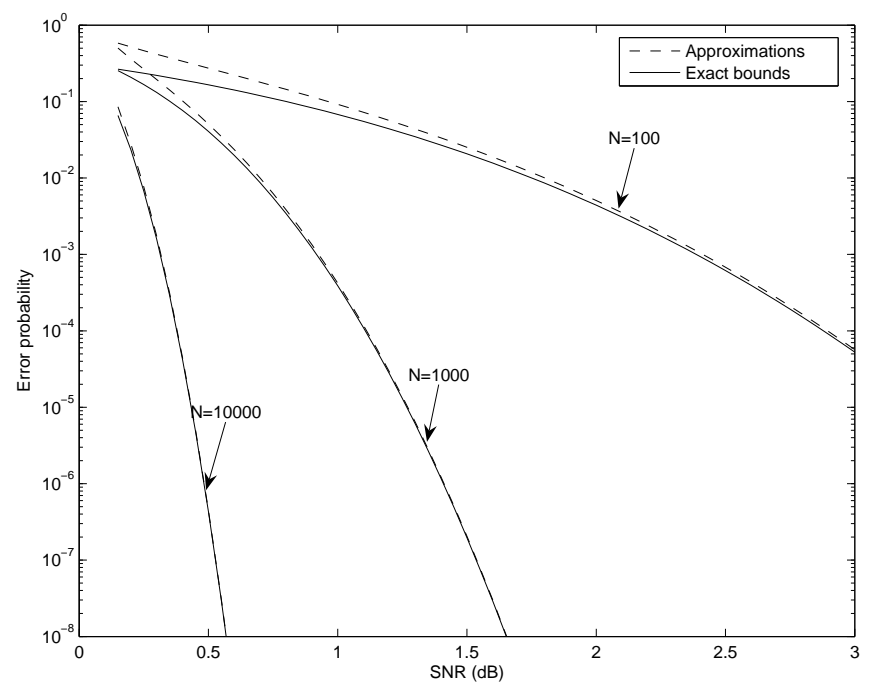

Fig. 1. Comparison of the approximate and exact expressions for the SP59 bound (see Eq. (6) and Thm. 4, respectively). The examined block lengths are $N=100,1000$ and 10,000 for a rate of 0.5 bits per ch. use per dimension.

\section{AN IMPRoved SPHERE-PACKING BOUND}

The original focus in the derivation of the SP67 bound [7] was the asymptotic analysis of the error probability for optimal codes when the block length tends to infinity. For this reason, the SP67 bound fails to provide informative results for codes of small to moderate block lengths. In [8], Valembois and Fossorier revisit the derivation of the SP67 bound, this time focusing on the error-probability of finite length codes. A new sphere-packing bound is derived in [8] which dramatically improves the tightness of the SP67 bound for codes with short to moderate block lengths; the new bound also extends the validity of the bound to memoryless channels with discrete input and continuous output. This improvement motivated us to investigate the possibility of further enhancing the tightness of the sphere-packing technique. In [10], [11], we derive the ISP bound which improves the tightness of the SP67 and Valembois-Fossorier bounds. Under a mild condition, the ISP bound is valid for general discrete-time memoryless channels. The new bound is applied to M-ary PSK block coded modulation over the AWGN channel and its tightness is compared with the SP59 bound which is valid for any set of equal energy signals over the AWGN channel.

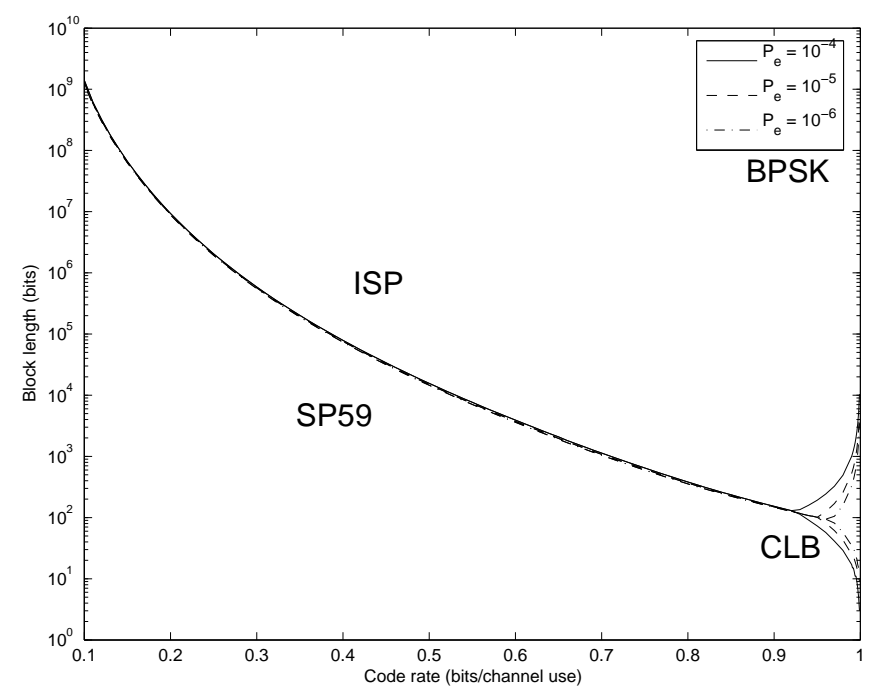

Fig. 2. Regions in the two-dimensional space of code rate and block length where a bound is better than the two others for three different targets of block error probability $\left(P_{\mathrm{e}}\right)$. The figure compares the tightness of the 1959 spherepacking (SP59) bound of Shannon [6], the improved sphere-packing (ISP) bound [10], and the capacity-limit bound (CLB). The plot refers to BPSK modulated signals whose transmission takes place over the AWGN channel, and the considered code rates lie in the range between 0.1 and $1 \frac{\text { bits }}{\text { channel use }}$.

Fig. 2 presents regions of code rates and block lengths for which the ISP bound outperforms the SP59 bound and the capacity-limit bound (CLB); it refers to BPSK modulated signals transmitted over the AWGN and considers block error probabilities of $10^{-4}, 10^{-5}$ and $10^{-6}$. It is reflected from this figure that for any rate $0<R<1$, there exists a block length $N(R)$ such that the ISP bound outperforms the SP59 bound for block lengths larger than $N(R)$ (the same property also holds for the Valembois-Fossorier bound, but that the value of $N(R)$ is significantly larger in the latter case). It is also observed that the value $N(R)$ is monotonically decreasing with $R$, and it approaches infinity as we let $R$ tend to zero. For relatively low code rates the SP59 bound outperforms the ISP bound for 
block lengths of interest in modern communication systems. For a further discussion and additional numerical results, the reader is referred to [10], [11].

\section{SUMMARY}

This paper presents an algorithm which performs the calculation of Shannon's 1959 sphere-packing (SP59) bound [6] in the logarithmic domain. This algorithm circumvents the numerical difficulties associated with a previous algorithm, suggested in [8], which performs the calculation in the probability domain. The new algorithm is used to calculate the bound for moderate to large block lengths, and to study the tightness of the asymptotic approximations in [6]. This work is part of a more general study of sphere-packing bounds [10]. A second part of this study is the derivation of an improved sphere-packing (ISP) bound which applies to general memoryless channels (the bound was recently presented in [11]), and the application of this bound to M-ary PSK block coded modulation over the AWGN channel. This allows to compare the tightness of the SP59 and ISP bounds for different rates, block lengths and modulation parameters (see Fig. 2, and for more details the reader is referred to [10], [11]). A comprehensive tutorial on sphere-packing bounds is given in [5, Chapter 5].

\section{APPENDIX}

PROOF OF PROPOSITION 1

From the definition of $f_{N}$ in (2), it follows that

$$
\begin{aligned}
f_{N}(x) & =\frac{1}{2^{\frac{N-1}{2}} \Gamma\left(\frac{N+1}{2}\right)} \int_{0}^{\infty} z^{N-1} \exp \left(-\frac{z^{2}}{2}+z x\right) d z \\
& =\frac{e^{\frac{x^{2}}{2}}}{2^{\frac{N-1}{2}} \Gamma\left(\frac{N+1}{2}\right)} \int_{0}^{\infty} z^{N-1} \exp \left(-\frac{(z-x)^{2}}{2}\right) d z \\
& =\frac{e^{\frac{x^{2}}{2}}}{2^{\frac{N-1}{2}} \Gamma\left(\frac{N+1}{2}\right)} \int_{-x}^{\infty}(u+x)^{N-1} \exp \left(-\frac{u^{2}}{2}\right) d u
\end{aligned}
$$

From the binomial formula, we get

$$
f_{N}(x)=\frac{e^{\frac{x^{2}}{2}}}{2^{\frac{N-1}{2}} \Gamma\left(\frac{N+1}{2}\right)} \sum_{j=0}^{N-1}\left[\left(\begin{array}{c}
N-1 \\
j
\end{array}\right) x^{N-1-j} \int_{-x}^{\infty} u^{j} \exp \left(-\frac{u^{2}}{2}\right) d u\right] .
$$

We now examine the integral in the RHS of (A.1). From symmetry (anti-symmetry) considerations of the integrand for even (odd) values of $j$, we get that

$$
\begin{aligned}
& \int_{-x}^{\infty} u^{j} \exp \left(-\frac{u^{2}}{2}\right) d u \\
& =\int_{0}^{\infty} u^{j} \exp \left(-\frac{u^{2}}{2}\right) d u+(-1)^{j} \int_{0}^{x} u^{j} \exp \left(-\frac{u^{2}}{2}\right) d u \\
& \stackrel{(a)}{=} \int_{0}^{\infty}(2 t)^{\frac{j-1}{2}} e^{-t} d t+(-1)^{j} \int_{0}^{\frac{x^{2}}{2}}(2 t)^{\frac{j-1}{2}} e^{-t} d t \\
& =2^{\frac{j-1}{2}} \int_{0}^{\infty} t^{\frac{j-1}{2}} e^{-t} d t \cdot\left[1+(-1)^{j} \frac{\int_{0}^{\frac{x^{2}}{2}} t^{\frac{j-1}{2}} e^{-t} d t}{\int_{0}^{\infty} t^{\frac{j-1}{2}} e^{-t} d t}\right] \\
& =2^{\frac{j-1}{2}} \Gamma\left(\frac{j+1}{2}\right)\left[1+(-1)^{j} \tilde{\gamma}\left(\frac{x^{2}}{2}, \frac{j+1}{2}\right)\right]
\end{aligned}
$$

where $(a)$ follows by substituting $t \triangleq \frac{u^{2}}{2}$, and the functions $\Gamma$ and $\tilde{\gamma}$ are defined in (9) and (10), respectively. Substituting the last equality in (A.1) and noting that

$$
\left(\begin{array}{c}
N-1 \\
j
\end{array}\right)=\frac{\Gamma(N)}{\Gamma(N-j) \Gamma(j+1)}, \quad N \in \mathbb{N}, j \in\{0,1, \ldots, N-1\}
$$

gives

$$
\begin{aligned}
f_{N}(x) \stackrel{(a)}{=} & \sum_{j=0}^{N-1}\left\{\frac{e^{\frac{x^{2}}{2}}}{\Gamma(N-j)} \frac{2^{N-1} \Gamma\left(\frac{N}{2}\right)}{\sqrt{\pi}} \frac{2^{-j} \sqrt{\pi}}{\Gamma\left(\frac{j}{2}+1\right)} \frac{x^{N-1-j}}{2^{\frac{N-j}{2}}}\right. \\
& \left.\cdot\left[1+(-1)^{j} \tilde{\gamma}\left(\frac{x^{2}}{2}, \frac{j+1}{2}\right)\right]\right\} \\
\stackrel{(b)}{=} & \sum_{j=0}^{N-1} \exp (d(N, j, x))
\end{aligned}
$$

where $(a)$ follows from the equality

$$
\Gamma(2 u)=\frac{2^{2 u-1}}{\sqrt{\pi}} \Gamma(u) \Gamma\left(u+\frac{1}{2}\right), \quad u \neq 0,-\frac{1}{2},-1,-\frac{3}{2}, \ldots
$$

and $(b)$ follows from the definition of $d(N, j, x)$ in (8).

\section{REFERENCES}

[1] S. Doliner, D. Divsalar and F. Pollara, "Code performance as a function of block size," Jet Propulsion Laboratory (JPL), TMO Progress Report 42-133, May 15, 1998. [Online] Available: http://tmo.jpl.nasa.gov/tmo/progress_report /42-133/133K.pdf.

[2] D. E. Lazic, Th. Beth and M. Calic, "How close are turbo codes to optimal codes ?," Proceedings of the International Symposium on Turbo Codes and Related Topics, pp. 192-195, Brest, France, 3-5 September 1997.

[3] D. E. Lazic, Th. Beth and S. Egner, "Constrained capacity of the AWGN channel," IEEE 1998 International Symposium on Information Theory (ISIT 1998), p. 237, Cambridge, MA, USA, 16-21 August, 1998.

[4] S. J. Macmullan nad O.M. Collins, "A comparison of known codes, randsom codes and the best codes," IEE Trans. on Information Theory, vol. 44, pp. 3009-3022, November 1998.

[5] I. Sason and S. Shamai, Performance Analysis of Linear Codes under Maximum-Likelihood Decoding: A Tutorial," Foundations and Trends in Communications and Information Theory, vol. 3, no. 1-2, pp. 1-222, NOW Publishers, Delft, the Netherlands, July 2006.

[6] C. E. Shannon, "Probability of error for optimal codes in a Gaussian channel," Bell System Technical Journal, vol. 38, pp. 611-656, May 1959.

[7] C. Shannon, R. Gallager and E. Berlekamp,"Lower bounds to error probability for decoding on discrete memoryless channels," Information and Control, vol. 10, Part 1: pp. 65-103, and Part 2: pp. 522-552, February/May 1967.

[8] A. Valembois and M. Fossorier, "Sphere-packing bounds revisited for moderate block length," IEEE Trans. on Information Theory, vol. 50, pp. 2998-3014, Decemeber 2004.

[9] L. Wei, "Near-optimum serial concatenation of single-parity codes with convolutional codes," IEE Proceedings on Communications, vol. 152, no. 4, pp. 397-403, August 2005.

[10] G. Wiechman and I. Sason, "Improved sphere-packing bound targeting codes of short to moderate block lengths and applications," submitted to IEEE Trans. on Information Theory, August 2006. [Online] Available: http: //arxiv.org/abs/cs. IT/0608042.

[11] —, "An improved sphere-packing bound targeting codes of short to moderate block lengths and applications," (invited paper) presented in the 44th Allerton Conference on Communication, Control and Computing, Monticello, Illinois, USA, 27-29 September 2006. 